\title{
In vitro ability of Staphylococcus aureus isolates from bacteraemic patients with and without metastatic complications to invade vascular endothelial cells
}

Correspondence

Hong Bin Kim

hbkimmd@snu.ac.kr
Received 7 June 2006 Accepted 25 June 2007

\author{
Wan Beom Park, ${ }^{1}$ Sung Han Kim, ${ }^{1}$ Cheol-in Kang, ${ }^{1}$ Jae Hyun Cho, ${ }^{1}$ \\ Ji Whan Bang, ${ }^{1}$ Kyoung Wha Park, ${ }^{1} \dagger$ Yeong Seon Lee, ${ }^{2}$ Nam Joong Kim, ${ }^{1}$ \\ Myoung-don Oh, ${ }^{1}$ Hong Bin $\mathrm{Kim}^{1} \ddagger$ and Kang Won Choe ${ }^{1}$
${ }^{1}$ Department of Internal Medicine, Seoul National University College of Medicine, Seoul 110-744, Republic of Korea
${ }^{2}$ National Institute of Health, Korea Center for Diseases Control and Prevention, Seoul 122-701, Republic of Korea

\begin{abstract}
Invasion of vascular endothelial cells is thought to be a critical step in the development of metastatic infections in patients with Staphylococcus aureus bacteraemia. This study was designed to evaluate the association between the ability to invade endothelial cells and metastatic infection by $S$. aureus. Patients with metastatic infection were identified among those with community-acquired $S$. aureus bacteraemia in a tertiary referral hospital. Patients with simple bacteraemia caused by $S$. aureus over the same period served as the control group. The ability of each clinical isolate to invade endothelial cells was evaluated by counting the number of intracellular organisms $1 \mathrm{~h}$ after inoculation onto human umbilical vein endothelial cells in vitro. The cytotoxic activity of intracellular $S$. aureus was determined $24 \mathrm{~h}$ after internalization, and expressed as the percentage of cells killed. The clinical isolates varied in invasiveness and cytotoxicity. The median invasiveness, relative to $S$. aureus reference strain ATCC 29213, was $145 \%$ in the cases $(n=10)$ [interquartile range (IQR) 103-160] and $153 \%$ (IQR 111-173) in the controls $(n=11 ; P=0.44)$. The median cytotoxicity was $59.4 \%$ (IQR $47-68$ ) in the cases and $65.2 \%$ (IQR 50-74) in the controls $(P=0.44)$. Differences in the ability of $S$. aureus to invade and destroy vascular endothelial cells in vitro were not associated with the development of metastatic complications in patients with $S$. aureus bacteraemia. This implies that the invasiveness and toxicity of $S$. aureus for endothelial cells may not be major determinants of metastatic infection.
\end{abstract}

\section{INTRODUCTION}

Staphylococcus aureus is an important cause of severe community-acquired and nosocomial bacteraemia (Weinstein et al., 1997). A major complication of S. aureus bacteraemia is the development of metastatic infection. The reported frequency of metastatic infection following $S$. aureus bacteraemia varies from 2 to $47 \%$ (Lautenschlager et al., 1993; Willcox et al., 1998; Mylotte et al., 1987; Nolan \& Beaty, 1976; Cunney et al., 1996; Finkelstein et al., 1984).

Abbreviations: HUVEC, human umbilical vein endothelial cell; IQR, interquartile range.

tPresent address: Department of Internal Medicine, Chonnam National University Hospital, Gwangju 501-757, Republic of Korea.

łPresent address: Department of Internal Medicine, Seoul National University Bundang Hospital, 300 Gumi-dong, Bundang-gu, Seongnamsi, Gyeonggi-do, 463-707, Republic of Korea.
The most common sites of metastatic foci include cardiac valve, bone and joints, lung, kidney, central nervous system and skin (Lautenschlager et al., 1993; Julander, 1985; Nolan \& Beaty, 1976; Mirimanoff \& Glauser, 1982). Metastatic complications in $S$. aureus bacteraemia are clinically important because they are associated with recurrent infection and grave outcome (Musher et al., 1994; Lautenschlager et al., 1993; Julander, 1985).

Although little is known about the pathogenesis of metastatic complications in S. aureus bacteraemia, it is thought that interaction between vascular endothelial cells and circulating bacteria is a first step in the extravasation of S. aureus (Lowy, 1998). Many studies have shown that $S$. aureus bacteria attach avidly to and invade vascular endothelial cells (Vercellotti et al., 1984; Ogawa et al., 1985). After invasion, the infected endothelial cells express Fc receptors and adhesion molecules, and then release 
interleukin-1, interleukin-6 and interleukin-8. This sequence of events facilitates the inflammatory process and contributes to the establishment of metastatic infection (Lowy, 1998). However, to the best of our knowledge, no study has demonstrated the clinical relevance of invasion of vascular endothelial cells to the development of metastatic infection in patients with $S$. aureus bacteraemia.

Internalized S. aureus can either survive for several days in the cytoplasm (Balwit et al., 1994) or induce the death of endothelial cells by apoptosis (Menzies \& Kourteva, 1998). Clinical isolates with increased cytotoxic activity produced higher lethality and more dissemination in a mouse septicaemia model (Krut et al., 2003). However, the clinical significance of the cytotoxicity of intracellular S. aureus remains unclear.

The purpose of the present study was to determine whether (1) the ability of $S$. aureus to invade vascular endothelial cells or (2) the cytotoxicity of intracellular S. aureus for vascular endothelial cells were determinants of metastatic infection in patients with $S$. aureus bacteraemia.

\section{METHODS}

Patients with and without metastatic infection. Cases of metastatic infection were identified among patients with community-acquired $S$. aureus bacteraemia at Seoul National University Hospital from January 1998 to December 2001. The control group consisted of patients with community-acquired and simple $S$. aureus bacteraemia over the same period.

The portal of entry was defined as the localized focus of $S$. aureus infection preceding bacteraemia, and any other foci were considered to represent metastatic infection (Lautenschlager et al., 1993). Community-acquired $S$. aureus bacteraemia was defined as the presence of a positive blood culture within $48 \mathrm{~h}$ of admission. A simple bacteraemic patient was defined as one with defervescence within 3 days of the onset of antibiotic treatment, and no evidence of metastatic complications.

Bacterial storage, growth and harvest. The clinical isolates and the S. aureus ATCC 29213 reference strain which had been stored in skimmed milk at $-70{ }^{\circ} \mathrm{C}$ were subcultured on blood agar. Fresh colonies were inoculated into brain heart infusion broth and grown overnight at $37^{\circ} \mathrm{C}$. Two hours before the experiment, overnight cultures were resuspended in fresh brain heart infusion broth and grown to mid-exponential phase. The bacteria were collected by centrifugation, washed three times in PBS, and resuspended in antibiotic-free EGM-2 medium (Cambrex). The suspensions were centrifuged at $75 \mathrm{~g}$ for $5 \mathrm{~min}$ to remove variable-sized aggregates (Van Belkum et al., 2002), and bacterial concentrations were measured spectrophotometrically at $620 \mathrm{~nm}$ and counted by plating serial dilutions on agar. Antibiotic susceptibility was determined by the disc diffusion method, as recommended by the CLSI (2005). PFGE was performed as described previously (Kim et al., 2006).

Preparation of endothelial cells. Human umbilical vein endothelial cells (HUVECs) were used in these experiments. They were grown to confluence in 24 -well plates at $37{ }^{\circ} \mathrm{C}$ in a humidified incubator with $5 \% \mathrm{CO}_{2}$, in antibiotic-free EGM-2 medium containing $5 \%$ fetal bovine serum and growth factors. All experiments were performed with cells that had been passaged between four and eight times.
Invasion assay. The invasion assay was performed to determine numbers of internalized bacteria, as described by Ogawa et al. (1985). Each well containing HUVECs was washed with antibiotic-free EGM2 medium prior to inoculation with bacteria. Bacterial suspension $\left(1 \mathrm{ml}\right.$ ), adjusted by optical density to $5 \times 10^{7}$ c.f.u. $\mathrm{ml}^{-1}$ (m.o.i. 250), was added to each well and incubated for $1 \mathrm{~h}$ at $37^{\circ} \mathrm{C}$ in $5 \% \mathrm{CO}_{2}$. The complementary study showed that the number of intracellular $S$. aureus was linearly correlated with the m.o.i., and that the difference in invasiveness between isolates was similar irrespective of m.o.i. under our experimental conditions (data not shown).

The plates were then washed twice with antibiotic-free EGM-2 medium and incubated at $37{ }^{\circ} \mathrm{C}$ for $20 \mathrm{~min}$ with antibiotic-free EGM-2 medium, which contained $10 \mu \mathrm{g}$ lysostaphin $\mathrm{ml}^{-1}$ (Sigma) to lyse extracellular staphylococci. The HUVECs were then released with trypsin, disrupted in hypotonic solution, and serial dilutions were plated on mannitol salt agar. Results are expressed as means \pm SEM of three independent experiments performed in duplicate.

Cytotoxicity assay. Prior to infection, HUVECs were washed with EGM-2 medium without antibiotics, and a total of $10^{7} \mathrm{~S}$. aureus were added per well (m.o.i. 50). Determination of the m.o.i. was based on our pilot study and a previous study (Krut et al., 2003). After $1 \mathrm{~h}$, the HUVECs were washed with antibiotic-free EGM-2 medium and incubated at $37{ }^{\circ} \mathrm{C}$ for $24 \mathrm{~h}$ with $10 \mu \mathrm{g}$ lysostaphin $\mathrm{ml}^{-1}$. The supernatants of the infected cultures were then carefully removed to preserve any detached cells, and adherent cells were harvested by standard trypsin treatment and combined with the supernatants. Viable cells were then counted by the trypan blue (Sigma) exclusion method (Bonifacio, 2000), and cytotoxicity was expressed as the percentage of dead cells. Three independent experiments were performed.

Statistical analysis. The Mann-Whitney U test and Fisher's exact test were used for comparison of continuous and categorical variables, respectively. $P<0.05$ was considered to be statistically significant. Statistical analysis was carried out using the SPSS software package.

\section{RESULTS}

\section{Patients and clinical isolates}

Fifty-one patients with community-acquired $S$. aureus bacteraemia were identified during the study period. Among these were 11 (22\%) cases of metastatic infection. Metastatic foci included cardiac valve, bone and joint, lung, central nervous system, and abdominal organs (Table 1). Eleven patients were selected as the control group, as described in Methods. The median age was 55.5 years (range 24-70) in the case group and 51 years (range 23-76) in the control group $(P=0.70)$. Sixty per cent of the case patients and $55 \%$ of the control patients were male $(P=1.00)$. The portal of entry was not identified in nine $(82 \%)$ of the patients with metastatic infection and in two $(18 \%)$ of the patients without metastatic infection $(P=0.01)$. An isolate from one patient in the case group was not available; therefore, 10 isolates from the case group and 11 from the control group were used in this study. All were meticillinsusceptible and PFGE did not show any evidence of outbreak in the community. Five patients in the case group and four in the control group were treated with anti-staphylococcal penicillin, and five patients in the case group and seven in the control group were treated with cephalosporin $(P=0.67)$. 
Table 1. Basal characteristics and site of infection in the $S$. aureus bacteraemic patients with or without metastatic infection

CRI, Catheter-related infection; IE, infective endocarditis; STI, soft tissue infection; CNS, central nervous system; PTBD, percutaneous biliary drainage.

\begin{tabular}{|c|c|c|c|c|c|c|}
\hline \multicolumn{4}{|c|}{ Bacteraemia with metastasis } & \multicolumn{3}{|c|}{ Simple bacteraemia } \\
\hline Age (years) & Sex & Portal of entry & Metastatic foci & Age (years) & Sex & Portal of entry \\
\hline 24 & M & CRI & Abdominal organ & 23 & $\mathrm{~F}$ & Pneumonia \\
\hline 34 & F & Unknown & IE, lung & 34 & $\mathrm{M}$ & STI \\
\hline 51 & $\mathrm{~F}$ & Unknown & CNS & 36 & $\mathrm{~F}$ & STI \\
\hline 51 & $\mathrm{~F}$ & Unknown & Osteomyelitis & 43 & $\mathrm{M}$ & Unknown \\
\hline 55 & M & STI & Lung & 47 & $\mathrm{~F}$ & CRI \\
\hline 56 & M & Unknown & $\begin{array}{l}\text { IE, CNS, abdominal } \\
\text { organ }\end{array}$ & 51 & $\mathrm{~F}$ & PTBD cholangitis \\
\hline 56 & M & Unknown & IE, CNS & 58 & M & STI \\
\hline $59^{*}$ & M & Unknown & Lung & 60 & $\mathrm{~F}$ & Unknown \\
\hline 64 & M & Unknown & IE, CNS & 62 & $\mathrm{M}$ & Wound infection \\
\hline 69 & M & Unknown & Osteomyelitis & 68 & $\mathrm{M}$ & STI \\
\hline 70 & $\mathrm{~F}$ & Unknown & Psoas abscess & 76 & $\mathrm{M}$ & Pneumonia \\
\hline
\end{tabular}

${ }^{*}$ An isolate from this patient was not available.

\section{Invasion assay}

The clinical S. aureus isolates varied in their ability to invade endothelial cells (Fig. 1). The median invasiveness, relative to the reference strain ATCC 29213, was $145 \%$ [interquartile range (IQR) 103-160] in the cases with metastatic infection and $153 \%$ (IQR 111-173) in the control patients without metastatic infection (Fig. 1). There was no significant difference in invasiveness between the case and control patients $(P=0.44)$.

\section{Cytotoxicity assay}

The cytotoxic activity of the clinical isolates varied considerably (Fig. 2). The median cytotoxicity was $59 \%$ (IQR 47-68) in the case group and $65 \%$ (IQR 50-74) in the control group. No statistically significant differences between the two groups were observed $(P=0.44)$.

The isolates from the patients without metastatic infection were also isolated from blood culture; therefore, they may have been more virulent than the colonizers from healthy carriers. We therefore performed cytotoxicity assays with an additional 10 isolates from healthy carriers. Colonizers from the healthy carriers had a median cytotoxicity of $55 \%$ (IQR 51-70), which was not statistically different from that of the clinical isolates $(P=0.87)$.

\section{DISCUSSION}

We demonstrated that although the clinical S. aureus isolates varied widely in their ability to invade endothelial cells, the isolates from patients with simple bacteraemia

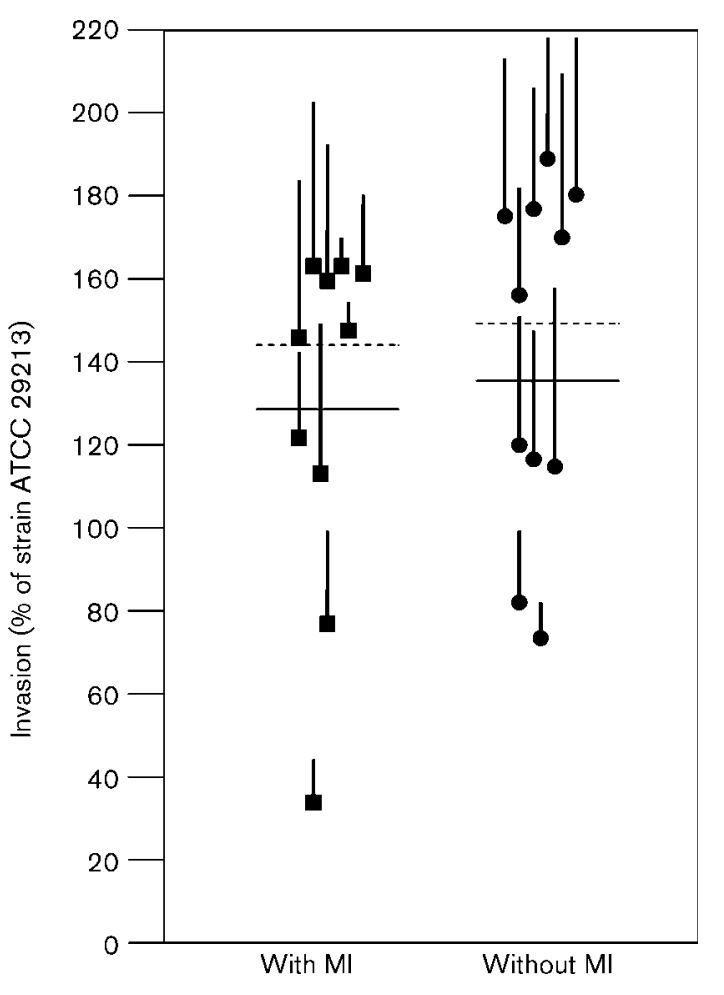

Fig. 1. Invasiveness of $S$. aureus clinical isolates from patients with or without metastatic infection (MI). The results are means \pm SEM of three independent experiments, expressed as invasiveness relative to strain ATCC 29213. Solid horizontal lines indicate the mean invasiveness of each group; dotted horizontal lines indicate the median invasiveness of each group. 


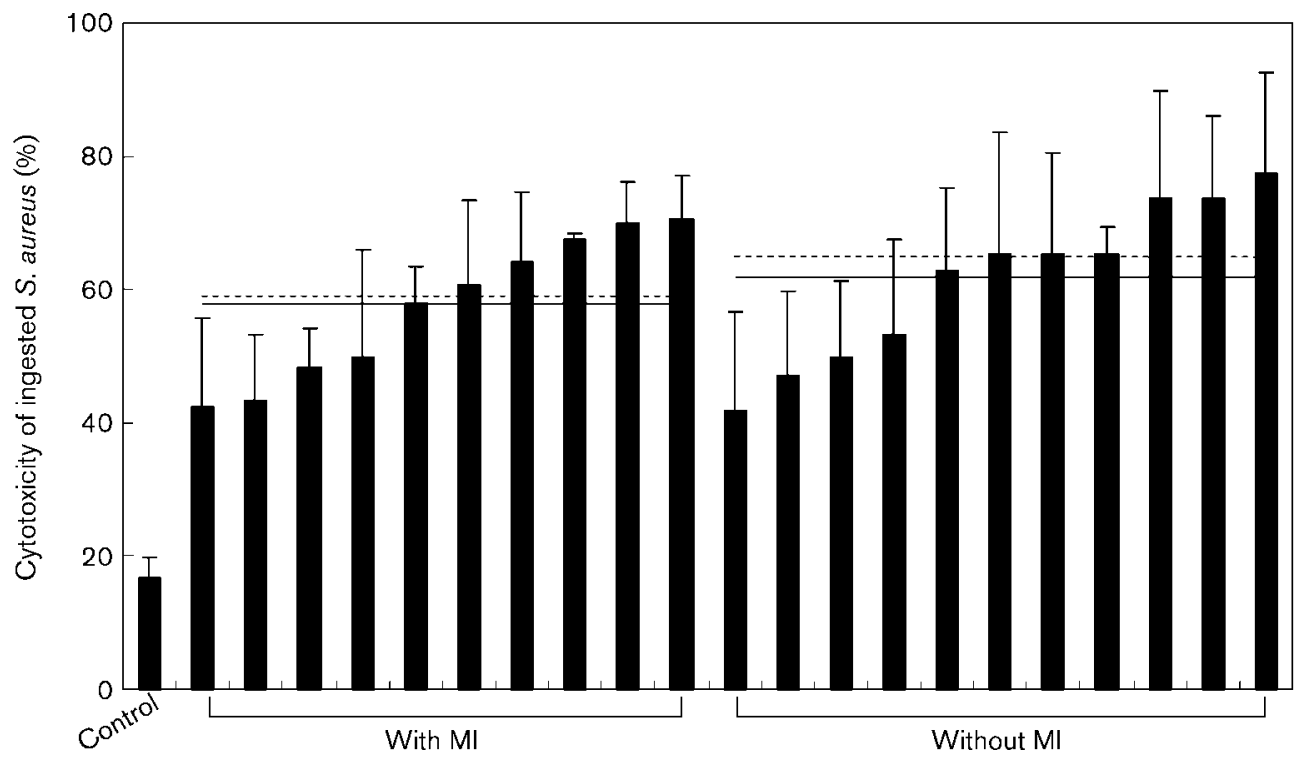

Fig. 2. Cytotoxicity of $S$. aureus isolates from patients with and without metastatic infection (MI). The percentage cell death was measured $24 \mathrm{~h}$ after infection. The results are expressed as the means \pm SEM of three independent experiments. Control denotes the percentage of dead cells in cultures not exposed to any bacteria. Solid horizontal lines indicate the mean cytotoxicity of each group; dotted horizontal lines indicate the median cytotoxicity of each group.

were as invasive as those from patients with metastatic complications. Since the bacteria must exit the infected cells and enter the subendothelial space in order to disseminate infection to other tissues, we also compared the cytotoxicity of the internalized S. aureus. Again, isolates from the patients without metastatic infection had equal ability to those from metastatic infections to kill the endothelial cells after internalization. Furthermore, colonizers from healthy carriers displayed similar cytotoxicity.

These data strongly suggest that some factor(s) other than those that permit the organism to invade and kill endothelial cells is needed for the development of a metastatic infection. Possibly, S. aureus contains either a molecule permitting adhesion to specific tissue sites (Marriott et al., 2005) or a toxin that can cause tissue injury (Fowler et al., 2005), or possesses a mechanism for evading the host immune system (Lowy, 1998). It is also possible that $S$. aureus extravasates via the intercellular space. Palmqvist et al. (2005) have shown that a gene knockout of $S$. aureus LS-1 which lacks fibronectin-binding protein gives rise to spontaneous arthritis, despite the fact that the fibronectin-binding protein is indispensable for invasion of endothelial cells (Peacock et al., 1999). It seems likely, therefore, that metastatic infection can develop without direct invasion of vascular endothelial cells. We suggest that host factors or host-pathogen interactions may be more important for the development of metastatic infection than are strictly microbial factors, because in a mouse bacteraemia model, we did not detect any difference in dissemination to internal organs in clinical isolates from patients with and without metastatic infection (data not shown).
Host factors that increase rates of metastatic seeding during $S$. aureus bacteraemia include: (1) an unknown primary focus; (2) community acquisition; and (3) S. aureus bacteraemia not associated with removable foci (Lautenschlager et al., 1993; Finkelstein et al., 1984; Hedstrom \& Christensson, 1983; Fowler et al., 2005). The present study also revealed an association between patients with metastatic infection and unknown primary focus. Perhaps S. aureus specifically responsible for communityacquired infection may have an unknown virulence factor that facilitates metastatic infection, given that patients from the community display relatively intact immunity in the usual clinical setting. Alternatively, community-acquired bacteraemia may be associated with persistent bacteraemia. The above-cited clinical studies support the hypothesis that bacteraemia of long duration is linked to metastatic infection. However, in this study, we were unable to examine this factor because the duration of bacteraemia prior to admission could not be established.

We confined the study population to patients with community-acquired S. aureus bacteraemia because: (1) patients with nosocomial $S$. aureus bacteraemia usually have severe immune defects that override other pathogenic virulence factors in the development of metastatic infection; and (2) community-acquired S. aureus may possess as-yet-unknown virulence factors that can promote the invasion of vascular endothelial cells.

The variable frequency of metastatic infection in earlier studies may have been due to variation in the methods of detection; for instance, the use of echocardiography to detect endocarditis in some cases (Ringberg et al., 2000). In 
the present study, to minimize the possibility of overlooking subclinical metastatic infection in the control group, we excluded those patients whose fever lasted $>3$ days after the initiation of antibiotic treatment, because a delay in response suggests the existence of undetected metastatic foci.

There are many methods for counting intracellular organisms, such as fluorescence assays and direct visual methods (Beekhuizen et al., 1997; Krut et al., 2003). In the present study, we chose the approach of plating and colony counting because it yields numbers of live organisms, and dead or dying organisms do not cause metastatic infection.

This study has several limitations. First of all, conditions of flow in vivo may influence the interaction of $S$. aureus with vascular endothelial cells, and endothelial receptors may show differential adhesion under shear flow conditions, with rolling and static receptors (McCormick et al., 1997). With respect to in vitro experiments and in vivo infection, there may be marked differences in expression of genes associated with virulence, such as that encoding fibronectin-binding protein, a major mediator of non-professional phagocyte invasion (Menzies, 2003). Finally, the number of clinical strains used in this study may not be sufficient to detect any subtle differences between each group. To prove the difference of invasiveness in $80 \%$ power and 0.05 alpha error, the isolates from the case group should have been $>30 \%$ more invasive than those from the control group.

In summary, clinical $S$. aureus isolates from patients with simple bacteraemia were as effective at invasion of vascular endothelial cells and induction of cell death as were isolates from patients with metastatic infection. This finding suggests the importance of host factors or as-yet-unidentified virulence factors other than invasiveness or cytotoxicity of $S$. aureus for vascular endothelial cells, which result in metastatic complications in patients with $S$. aureus bacteraemia.

\section{ACKNOWLEDGEMENTS}

This work was presented at the 43rd annual meeting of the Infectious Diseases Society of America, San Francisco, CA, USA, 2005. The work was supported by grant no. 02-05-026 from the research fund of Seoul National University Bundang Hospital.

\section{REFERENCES}

Balwit, J. M., van Langevelde, P., Vann, J. M. \& Proctor, R. A. (1994). Gentamicin-resistant menadione and hemin auxotrophic Staphylococcus aureus persist within cultured endothelial cells. J Infect Dis 170, 1033-1037.

Beekhuizen, H., van de Gevel, J. S., Olsson, B., van Benten, I. J. \& van Furth, R. (1997). Infection of human vascular endothelial cells with Staphylococcus aureus induces hyperadhesiveness for human monocytes and granulocytes. J Immunol 158, 774-782.

Bonifacio, J. S. (2000). Current Protocols in Cell Biology. New York: Wiley.

CLSI (2005). Performance Standards for Antimicrobial Disk Susceptibility Tests. Wayne, PA: Clinical and Laboratory Standards Institute.
Cunney, R. J., McNamara, E. B., alAnsari, N. \& Smyth, E. G. (1996). Community and hospital acquired Staphylococcus aureus septicaemia: 115 cases from a Dublin teaching hospital. J Infect 33, 11-13.

Finkelstein, R., Sobel, J. D., Nagler, A. \& Merzbach, D. (1984). Staphylococcus aureus bacteremia and endocarditis: comparison of nosocomial and community-acquired infection. J Med 15, 193-211.

Fowler, V. G., Jr, Justice, A., Moore, C., Benjamin, D. K., Jr, Woods, C. W., Campbell, S., Reller, L. B., Corey, G. R., Day, N. P. \& Peacock, S. J. (2005). Risk factors for hematogenous complications of intravascular catheter-associated Staphylococcus aureus bacteremia. Clin Infect Dis 40, 695-703.

Hedstrom, S. A. \& Christensson, B. (1983). Staphylococcus aureus septicaemia and endocarditis at the University Hospital in Lund 1976-1980. Scand J Infect Dis Suppl 41, 38-48.

Julander, I. (1985). Unfavourable prognostic factors in Staphylococcus aureus septicemia and endocarditis. Scand J Infect Dis 17, 179-187.

Kim, H. B., Lee, Y. S., Kim, B. S., Cha, J. O., Kwon, S. U., Lee, H. J., Suh, J. T., Rheem, I., Kim, J. M. \& other authors (2006). Prevalence and clinical implications of Staphylococcus aureus with a vancomycin MIC of $4 \mu \mathrm{g} / \mathrm{ml}$ in Korea. Microb Drug Resist 12, 33-38.

Krut, O., Utermohlen, O., Schlossherr, X. \& Kronke, M. (2003). Strain-specific association of cytotoxic activity and virulence of clinical Staphylococcus aureus isolates. Infect Immun 71, 2716-2723.

Lautenschlager, S., Herzog, C. \& Zimmerli, W. (1993). Course and outcome of bacteremia due to Staphylococcus aureus: evaluation of different clinical case definitions. Clin Infect Dis 16, 567-573.

Lowy, F. D. (1998). Staphylococcus aureus infections. N Engl J Med 339, 520-532.

Marriott, I., Gray, D. L., Rati, D. M., Fowler, V. G., Jr, Stryjewski, M. E., Levin, L. S., Hudson, M. C. \& Bost, K. L. (2005). Osteoblasts produce monocyte chemoattractant protein-1 in a murine model of Staphylococcus aureus osteomyelitis and infected human bone tissue. Bone 37, 504-512.

McCormick, C. J., Craig, A., Roberts, D., Newbold, C. I. \& Berendt, A. R. (1997). Intercellular adhesion molecule-1 and CD36 synergize to mediate adherence of Plasmodium falciparum-infected erythrocytes to cultured human microvascular endothelial cells. J Clin Invest 100, 2521-2529.

Menzies, B. E. (2003). The role of fibronectin binding proteins in the pathogenesis of Staphylococcus aureus infections. Curr Opin Infect Dis 16, 225-229.

Menzies, B. E. \& Kourteva, I. (1998). Internalization of Staphylococcus aureus by endothelial cells induces apoptosis. Infect Immun 66, 5994-5998.

Mirimanoff, R. O. \& Glauser, M. P. (1982). Endocarditis during Staphylococcus aureus septicemia in a population of non-drug addicts. Arch Intern Med 142, 1311-1313.

Musher, D. M., Lamm, N., Darouiche, R. O., Young, E. J., Hamill, R. J. \& Landon, G. C. (1994). The current spectrum of Staphylococcus aureus infection in a tertiary care hospital. Medicine (Baltimore) 73, 186-208.

Mylotte, J. M., McDermott, C. \& Spooner, J. A. (1987). Prospective study of 114 consecutive episodes of Staphylococcus aureus bacteremia. Rev Infect Dis 9, 891-907.

Nolan, C. M. \& Beaty, H. N. (1976). Staphylococcus aureus bacteremia. Current clinical patterns. Am J Med 60, 495-500.

Ogawa, S. K., Yurberg, E. R., Hatcher, V. B., Levitt, M. A. \& Lowy, F. D. (1985). Bacterial adherence to human endothelial cells in vitro. Infect Immun 50, 218-224.

Palmqvist, N., Foster, T., Fitzgerald, J. R., Josefsson, E. \& Tarkowski, A. (2005). Fibronectin-binding proteins and fibrinogen-binding clumping 
factors play distinct roles in staphylococcal arthritis and systemic inflammation. J Infect Dis 191, 791-798.

Peacock, S. J., Foster, T. J., Cameron, B. J. \& Berendt, A. R. (1999). Bacterial fibronectin-binding proteins and endothelial cell surface fibronectin mediate adherence of Staphylococcus aureus to resting human endothelial cells. Microbiology 145, 3477-3486.

Ringberg, H., Thoren, A. \& Lilja, B. (2000). Metastatic complications of Staphylococcus aureus septicemia. To seek is to find. Infection 28, 132-136.

Van Belkum, A., Kools-Sijmons, M. \& Verbrugh, H. (2002). Attachment of Staphylococcus aureus to eukaryotic cells and experimental pitfalls in staphylococcal adherence assays: a critical appraisal. J Microbiol Methods 48, 19-42.
Vercellotti, G. M., Lussenhop, D., Peterson, P. K., Furcht, L. T., McCarthy, J. B., Jacob, H. S. \& Moldow, C. F. (1984). Bacterial adherence to fibronectin and endothelial cells: a possible mechanism for bacterial tissue tropism. J Lab Clin Med 103, 34-43.

Weinstein, M. P., Towns, M. L., Quartey, S. M., Mirrett, S., Reimer, L. G., Parmigiani, G. \& Reller, L. B. (1997). The clinical significance of positive blood cultures in the 1990s: a prospective comprehensive evaluation of the microbiology, epidemiology, and outcome of bacteremia and fungemia in adults. Clin Infect Dis 24, 584-602.

Willcox, P. A., Rayner, B. L. \& Whitelaw, D. A. (1998). Communityacquired Staphylococcus aureus bacteraemia in patients who do not abuse intravenous drugs. QJM 91, 41-47. 